\title{
Editorial
}

\section{New Trends in Asymmetric Catalysis}

\author{
Rosaria Schettini (D) and Giorgio Della Sala *(D) \\ Dipartimento di Chimica e Biologia "Adolfo Zambelli" /DCB, Università di Salerno, 84084 Salerno, Italy; \\ schettinirosaria89@gmail.com \\ * Correspondence: gdsala@unisa.it
}

Citation: Schettini, R.; Della Sala, G. New Trends in Asymmetric Catalysis. Catalysts 2021, 11, 306. https:// doi.org/10.3390/catal11030306

Received: 22 February 2021

Accepted: 24 February 2021

Published: 26 February 2021

Publisher's Note: MDPI stays neutral with regard to jurisdictional claims in published maps and institutional affiliations.

Copyright: (c) 2021 by the authors. Licensee MDPI, Basel, Switzerland. This article is an open access article distributed under the terms and conditions of the Creative Commons Attribution (CC BY) license (https:// creativecommons.org/licenses/by/ $4.0 /)$.
As far back as the mid-nineteenth century, the studies of Louis Pasteur brought to light the essential role of molecular chirality in biology. Since then, organic chemists, biologists and physicians have become more and more aware of the close correlation between the biological activity and chirality of organic compounds, resulting from mediation by chiral biological receptors. This assumption, corroborated by the drawbacks associated with the use of racemic materials (the case of thalidomide is exemplary) led, through the second half of the twentieth century, to a rapid development of methods to selectively obtain the eutomer of a natural product or a synthetic analog for many applications, including pharmaceuticals, food chemicals, health care products and materials. The early efforts in the asymmetric synthesis field were based on the racemic resolution methods and later on the use of enantiopure stoichiometric reagents and chiral auxiliaries. However, the real breakthrough took place in the 1970s and 1980s with the emergence of asymmetric catalysis, marked out by the outstanding achievements by Knowles and Noyori in enantioselective hydrogenation, by Sharpless in enantioselective epoxidation and the subsequent implementation of several industrial processes. Since in principle a single molecule of chiral catalyst can generate tens, hundreds or even thousands of molecules of enantioenriched product, which Noyori referred to as multiplication of chirality, asymmetric catalysis can rightly be considered the highest evolution of asymmetric synthesis. The end of the twentieth century has seen an explosion of methods based on the combination of newly designed chiral organic ligands and metal centers, as well as a rapid growth of asymmetric biocatalysis. The beginning of this new century marked a revival of the use of small organic chiral molecules as catalysts. The advantages of organocatalysis in terms of environmental impact, low costs and simplicity of reaction set-up have indeed led to an impressive development of this strategy in the last two decades. To this day, asymmetric catalysis, founded on three main pillars-metal catalysis, organocatalysis and biocatalysis-is undoubtedly the most powerful tool for preparing enantioenriched products, and the ever-increasing research effort made in this field is therefore not surprising.

Although a large volume of work is still devoted to designing novel and more efficient chiral ligands and organocatalysts, and to expanding the scope of accessible enantioselective transformations, more and more attention is being paid to developing more sustainable processes. Accordingly, techniques requiring lower environmental impact, waste reduction, lower energy cost and the use of cheap, readily available and recyclable reagents and catalysts are being pursued. In this respect, the articles collected in the present Special Issue focus on these new trends.

Proline is one of the smallest and most inexpensive and readily available chiral organocatalysts. Unfortunately, a major drawback is its poor solubility in most organic solvents, enabling good enantioselectivities and catalytic activities only in polar aprotic solvents, such as dimethylsulfoxide, dimethylformamide or acetonitrile, which are highboiling, toxic and expensive. The article by Quintavalla et al. provides a sustainable protocol for the proline-catalyzed asymmetric aldol reaction in methanol and water [1]. Unsatisfactory results were obtained by using pure solvents, since methanol was affected 
by poor stereocontrol, and water led to low reaction rates. The best compromise was achieved by using methanol-water mixtures.

Four review articles offer interesting overviews of some hot topics in asymmetric catalysis. The application of electrochemistry to enantioselective organic synthesis has recently received a renewed interest, being associated with mild reaction conditions and reduced energy consumption. The review of Margarita and Lundberg describes the recent advances in asymmetric metal-catalyzed and organocatalyzed electrosynthesis, reporting examples of $\mathrm{C}-\mathrm{H}$ and alkene functionalization, carboxylation and cross-coupling reactions, and the related mechanistic insight [2]. The review is divided into oxidative and reductive transformations, highlighting that asymmetric oxidative processes are more explored compared to reductive ones.

The asymmetric ring-opening of epoxides is an important method for the preparation of enantioenriched vicinal difunctionalized organic compounds. The review by Warnmark et al. [3] provides a comprehensive overview of the desymmetrizations of meso-epoxides and the kinetic resolution of racemic chiral epoxides, catalyzed by chiral metal-salen complexes, a privileged class of effective and easily tunable chiral catalysts. Throughout their discussion, the authors have stressed the recent progresses made, reducing the catalyst loading and preparing easily recyclable supported catalysts. Particularly relevant is the application of multi-metallic catalysts, which has enabled improved performances and further mechanistic insight. At the end of their review, the authors outline the possible future developments, with particular reference to the substrate scope expansion.

Sunlight is a one of the most available and renewable energy sources, and as such, its application in green organic synthesis is extremely attractive. From this perspective, asymmetric visible-light photocatalysis has experienced a remarkable growth in the last decade. Moreover, the mechanism of photocatalyzed redox reactions involves the formation of unusual radical intermediates, enabling synthetic transformations that are difficult to accomplish under conventional conditions. The review by Alemán et al. [4] summarizes the achievements of the last few years in the $\alpha$-functionalization of imine building blocks, both racemic and stereoselective, by means of visible-light photoredox catalysis. As outlined in the introduction, imines are versatile substrates under photoredox catalysis conditions, which can serve either as nucleophiles or electrophiles. The photocatalytic addition reactions of radicals to imines and reactions involving $\alpha$-aminoradicals, generated by the single-electron reduction of imines, are covered in different sections. However, as pointed out by the authors, despite the impressive advances made in this field, the asymmetric functionalization of imine bonds remains a formidable challenge.

N-heterocyclic carbenes (NHCs) are valuable Lewis base species largely employed in asymmetric catalysis. Their mode of action involves the generation of intermediates such as acyl anions, homoenolates, enolates and $\alpha, \beta$-unsaturated acylazolium equivalents, and their subsequent reaction with electrophiles. Recently, as illustrated in the review by Ren et al. [5], Lewis acids, in combination with chiral NHC catalysts, were proved to increase the reaction yields and enantioselectivity of many reactions, as well as decisively affect (often reversing) regio- and diastereoselectivities. The review examines several examples categorized according to the Lewis acid type involved.

In conclusion, this collection of publications well represents the progresses and latest trends in the constantly evolving area of asymmetric catalysis. We wish to thank the authors for their valuable contributions, and we hope that this Special Issue could be inspiring for many scholars active in this field.

Funding: This research received no external funding.

Conflicts of Interest: The authors declare no conflict of interest. 


\section{References}

1. Emma, M.G.; Tamburrini, A.; Martinelli, A.; Lombardo, M.; Quintavalla, A.; Trombini, C. A Simple and Efficient Protocol for Proline-Catalysed Asymmetric Aldol Reaction. Catalysts 2020, 10, 649. [CrossRef]

2. Margarita, C.; Lundberg, H. Recent Advances in Asymmetric Catalytic Electrosynthesis. Catalysts 2020, 10, 982. [CrossRef]

3. Lidskog, A.; Li, Y.; Wärnmark, K. Asymmetric Ring-Opening of Epoxides Catalyzed by Metal-Salen Complexes. Catalysts 2020, 10, 705. [CrossRef]

4. Garrido-Castro, A.F.; Maestro, M.C.; Alemán, J. $\alpha$-Functionalization of Imines via Visible Light Photoredox Catalysis. Catalysts 2020, 10, 562. [CrossRef]

5. Jia, Q.; Li, Y.; Lin, Y.; Ren, Q. The Combination of Lewis Acid with N-Heterocyclic Carbene (NHC) Catalysis. Catalysts 2019, 9 , 863. [CrossRef] 